CERN-TH 99-413

December 1999

\title{
Adjoint String Breaking in 4d SU(2) Yang-Mills Theory
}

\author{
Philippe de Forcrand ${ }^{1,2}$ and Owe Philipsen ${ }^{2}$ \\ ${ }^{1}$ ETH-Hönggerberg, CH-8093 Zürich, Switzerland \\ ${ }^{2}$ CERN-TH, CH-1211 Geneva 23, Switzerland
}

\begin{abstract}
We compute the static potential of adjoint sources in SU(2) Yang-Mills theory in four dimensions by numerical Monte Carlo simulations. Following a recent calculation in $2+1$ dimensions, we employ a variational approach involving string and gluelump operators and obtain clear evidence for string breaking and the saturation of the potential at large distances. For the string breaking scale we find $r_{b} \approx 1.25 \mathrm{fm}, 2.3 r_{0}$, or in units of the lightest glueball, $r_{b} m_{0++} \approx 9.7$. We furthermore resolve the first excitation of the flux-tube and observe its breaking as well. The result for $r_{b}$ is in remarkable quantitative agreement with the threedimensional one.
\end{abstract}

CERN-TH 99-413 


\section{Introduction}

The most essential property of QCD is confinement: the energy of a static quarkantiquark $Q \bar{Q}$ pair grows linearly with their separation $r$, because the colour flux between the two charges is negligible outside a thin tube, the string, whose energy grows in proportion to its length. This remains true up to a distance $r_{b}$ where the string breaks: the energy becomes sufficient to create a pair of mesons, $Q \bar{q}$ and $\bar{Q} q$, which can be taken arbitrarily far apart at no extra energy cost. The static potential thus levels off at a value $2 E(Q \bar{q})$. Similarly, the force between a pair of adjoint sources $Q_{a d j}$ is screened by gluons $g$, so that the adjoint string breaks, even in the absence of quarks, when a pair of "gluelumps" $Q_{a d j} g$ can be created. In numerical simulations of lattice QCD it has been far easier to observe the string itself than its breaking. In spite of extensive searches for colour screening [1], the evidence is still preliminary at best [2].

The essential reason lies in the projection properties of the Wilson loop, whose change from an area law to a perimeter law behaviour would signal string breaking. The difficulty with seeing the perimeter law in simulations stems from the fact that at $r>r_{b}$ the state with unbroken string between the sources is still an eigenstate of the Hamiltonian, albeit an excited one. By construction, the Wilson loop has good overlap with this state, which

remains the case also for $r>r_{b}$. If it also has poor overlap with the two-gluelump state, then for $r>r_{b}$ the latter, which is the true ground state, will only dominate the correlation function for very large correlation times $t$, which according to the definition of the potential should be taken to infinity for the loop to yield the ground state. In most practical simulations, the limit $t \rightarrow \infty$ is not realized, but $t$ is typically a few lattice spacings when the signal is lost in noise. If this happens before $t$ is large enough for the ground state to dominate the correlation function, the ground state will be missed and an excited state will be extracted instead, leading to the apparent conclusion that the string does not break [3].

A way to circumvent this difficulty is to supplement the calculation with an additional operator having good projection onto the two-gluelump state. This enables an unambiguous identification of the ground state also for $r>r_{b}$, even if the perimeter behaviour of the Wilson loop is hidden in noise. After a first attempt for the adjoint potential [4], this procedure has been first sucessful for the fundamental static potential of the $\mathrm{SU}(2)$ Higgs model in its confining phase in $(2+1)$ [5] as well as in $(3+1)$ [6] dimensions, where it clearly demonstrated the flattening of the potential as well as mixing between the Wilson loop and the broken string state in a very narrow region around 
$r_{b}$. The same findings were reported from simulations of the adjoint potential in $(2+1)$ dimensional Yang-Mills theory [7, 8].

In this work, we study string breaking in the adjoint potential of SU(2) Yang-Mills theory in four dimensions. In practical terms, the advantage over QCD is that no dynamical quarks need be considered. The price to pay is that the adjoint string tension is larger than the fundamental one, approximately by the ratio $8 / 3$ of the Casimirs, so that Wilson loops obeying an area law disappear more quickly in the statistical noise, which turned out to hamper the earlier study [4.

Section 2 describes our calculational method for on- and off-axis correlations. Section 3 presents our simulations and Section 4 our numerical results. Section 5 contains a summary and outlook.

\section{Calculational method}

We work with the Wilson action

$$
S[U]=\beta_{G} \sum_{x} \sum_{\mu<\nu}\left[1-\frac{1}{2} \operatorname{Tr} P_{\mu \nu}(x)\right],
$$

where $P_{\mu \nu}$ denotes a plaquette of links $U_{\mu}$ in the fundamental representation, $\beta_{G}=4 / g^{2}$ and $g$ is the bare gauge coupling. The links in the adjoint representation, $A_{\mu}^{a b}$, are related to the fundamental $U_{\mu}$ 's by

$$
A_{\mu}^{a b}(x)=\frac{1}{2} \operatorname{Tr}\left(\sigma^{a} U_{\mu}(x) \sigma^{b} U_{\mu}^{\dagger}(x)\right),
$$

where $\sigma^{a}$ are the Pauli matrices.

The standard operators to extract the static potential are rectangular Wilson loops with side lengths $r$ and $t$ in a spatial and the time direction, respectively. Physically, a Wilson line $S(x, y)$ is interpreted as colour flux propagating along that line from $x$ to $y$. Accordingly, a straight Wilson line in $t$-direction corresponds to a static colour source, whereas a Wilson line in $r$-direction may be used to describe a flux tube between $x$ and $y$. A rectangular Wilson loop then represents the correlation function of a string of length $r$ over a time interval $t$. To consider the case of adjoint sources, an adjoint Wilson loop is needed, which takes the form

$$
G_{S S}(r, t)=W_{\mathrm{adj}}(r, t)=|W(r, t)|^{2}-1
$$

where

$$
W(r, t)=\operatorname{Tr}\left[S(0, r \hat{\mathrm{j}}) S(r \hat{\mathrm{j}}, r \hat{\mathrm{j}}+t \hat{0}) S^{\dagger}(t \hat{0}, r \hat{\mathrm{j}}+t \hat{0}) S^{\dagger}(0, t \hat{0})\right]
$$


is the fundamental Wilson loop, $S(x, y)$ is a straight line of fundamental links connecting the sites $x$ and $y$, and $\hat{j}$ and $\hat{0}$ denote unit vectors in a spatial and the time direction respectively. The static potential in the adjoint representation is defined in terms of the exponential decay of the adjoint Wilson loop,

$$
V(r)=-\lim _{t \rightarrow \infty} \frac{1}{t} \ln \left[W_{\mathrm{adj}}(r, t)\right] .
$$

In the region of linear confinement the Wilson loop obeys an area law, whereas for distances beyond some breaking scale $r_{b}$ a perimeter law is expected. This perimeter behaviour for large separations $r$, however, has not been observed to date.

The correlation function for a bound state of a static adjoint colour source and a gluon field, called gluelump in the literature [9], is given by the non-local gauge-invariant operator

$$
\begin{aligned}
G_{G}(t) & =\left\langle\operatorname{Tr}\left(P(x) \sigma^{a}\right) S_{a d j}^{a b}(x, x+t \hat{0}) \operatorname{Tr}\left(P^{\dagger}(x+t \hat{0}) \sigma^{b}\right)\right\rangle \\
& =\left\langle\operatorname{Tr}\left[P(x) S(x, x+t \hat{0})\left(P^{\dagger}(x+t \hat{0})-P(x+t \hat{0})\right) S^{\dagger}(x, x+t \hat{0})\right]\right\rangle
\end{aligned}
$$

with the adjoint representation Wilson line

$$
S_{a d j}^{a b}(x, y)=\frac{1}{2} \operatorname{Tr}\left(\sigma^{a} S(x, y) \sigma^{b} S^{\dagger}(x, y)\right)
$$

Here $P(x)$ denotes the "clover-leaf" of all four plaquettes with the same orientation which emanate from the endpoint $x$ of the adjoint Wilson line, summed over the three spatial planes [4].

We now follow the procedure proposed in [4] and applied in three dimensions [8] to construct an operator projecting on two of these bound states at distance $r$ by

$$
\begin{aligned}
G_{G G}(r, t)= & \left\langle\operatorname{Tr}\left[P(0) S(0, t \hat{0})\left(P^{\dagger}(t \hat{0})-P(t \hat{0})\right) S^{\dagger}(0, t \hat{0})\right]\right. \\
& \left.\times \operatorname{Tr}\left[P(r \hat{\jmath}) S(r \hat{\jmath}, r \hat{\jmath}+t \hat{0})\left(P^{\dagger}(r \hat{\jmath}+t \hat{0})-P(r \hat{\jmath}+t \hat{0})\right) S^{\dagger}(r \hat{\jmath}, r \hat{\jmath}+t \hat{0})\right]\right\rangle .
\end{aligned}
$$

Finally, correlations between a string and a gluelump state, and vice versa, may be described by

$$
\begin{aligned}
G_{S G}(r, t)= & \left\langle\operatorname { T r } \left[\left(P^{\dagger}(t \hat{0})-P(t \hat{0})\right) S^{\dagger}(0, t \hat{0}) S(0, r \hat{\mathrm{j}}) S(r \hat{\mathrm{j}}, r \hat{\mathrm{j}}+t \hat{0})\right.\right. \\
& \left.\left.\times P(r \hat{\mathrm{j}}+t \hat{0}) S^{\dagger}(r \hat{\mathrm{j}}, r \hat{\mathrm{j}}+t \hat{0}) S^{\dagger}(0, r \hat{\mathrm{\jmath}}) S(0, t \hat{0})\right]\right\rangle, \\
G_{G S}(r, t)= & \left\langle\operatorname { T r } \left[\left(P^{\dagger}(0)-P(0)\right) S(0, t \hat{0}) S(t \hat{0}, r \hat{\mathrm{j}}+t \hat{0}) S^{\dagger}(r \hat{\mathrm{j}}, r \hat{\mathrm{j}}+t \hat{0})\right.\right. \\
& \left.\left.\times P(r \hat{\mathrm{j}}) S(r \hat{\mathrm{j}}, r \hat{\mathrm{j}}+t \hat{0}) S^{\dagger}(t \hat{0}, r \hat{\mathrm{\jmath}}+t \hat{0}) S^{\dagger}(0, t \hat{0})\right]\right\rangle .
\end{aligned}
$$


The static potential, its excitations and the mixing between gauge string and twogluelump state can then be extracted from measurements of the matrix correlator

$$
G(r, t)=\left(\begin{array}{cc}
G_{S S}(r, t) & G_{S G}(r, t) \\
G_{G S}(r, t) & G_{G G}(r, t)
\end{array}\right) .
$$

We also keep the single gluelump operator $G_{G}$ from (6) in our simulations, in order to check whether $G_{G G}$ defined in (8) indeed has a good projection onto a two-gluelump state, for which one expects $E_{G G} \simeq 2 E_{G}$.

A particular difficulty in observing string breaking comes from its suddenness. As the distance between the sources increases, the ground state switches abruptly from an unbroken string overlapping almost exclusively with the Wilson loop, to a broken string overlapping almost exclusively with two gluelumps. Mixing among the two states, which proves that one passes continuously from one ground state to the other, occurs only if the two states are very close in mass. This corresponds to a very narrow window of distances, which becomes elusive or entirely unobservable on a coarse lattice [4]. For this reason, we supplement our study of on-axis correlations as above with that of off-axis correlations, which offer far more discrete Euclidean distances. To avoid computing spatial parallel transporters from $x$ to $y$ as in eqs.(4,8,9) for off-axis separations, we instead fix the gauge and measure in a fixed gauge the generic adjoint correlator between two time-like "segments"

$$
G_{f i x}(r, t)=\left\langle\left|\operatorname{TrS}(0, \mathrm{t} \hat{0}) \mathrm{S}^{\dagger}(\mathrm{r} \hat{\mathrm{j}}, \mathrm{r} \hat{\mathrm{j}}+\mathrm{t} \hat{0})\right|^{2}-1\right\rangle \text {. }
$$

Any gauge which preserves the transfer matrix is acceptable. The choice of gauge will determine the projection of the above correlator onto the string or the two-gluelump state. To project onto the broken string, $G_{f i x} \sim G_{G G}$, we simply choose the gauge (defined up to an irrelevant Abelian rotation $\exp \left(\mathrm{i} \theta \sigma_{3}\right)$ ) which brings $F=\frac{1}{2 i}\left(P-P^{\dagger}\right)$ to the colour direction $\sigma_{3}$ at every point: $F^{1}(x)=F^{2}(x)=0 \forall x$. The required local gauge transformation can be determined and applied independently at each lattice site. To project onto the unbroken string, $G_{f i x} \sim G_{S S}$, we need a smooth gauge and choose the adjoint Coulomb gauge. Correspondingly, for the mixed elements $G_{S G}$ the gauges on the two timeslices in the correlator $G_{f i x}$ are different. In order to avoid possible systematic effects from lattice "Gribov" copies, we discard the usual implementation of Coulomb gauge on the lattice by iterative local extremization [10 in favour of the unambiguous prescription of Vink and Wiese [11]. Gauge-fixing is accomplished by rotating the two lowest-lying eigenvectors of the adjoint covariant $3 d$ Laplacian to direction $\sigma_{3}$ and into the plane $\left(\sigma_{1}, \sigma_{3}\right)$ respectively in colour space. This gauge condition corresponds to the Laplacian Center Gauge described in [12], with only the three spatial directions used for 
the Laplacian. It reduces to Coulomb gauge for the adjoint field in the continuum limit. It is unambiguous except when either of the lowest two eigenvalues of the Laplacian becomes degenerate, which signals a genuine (not a lattice artifact) Gribov copy. Such a degeneracy did not occur over the whole course of our simulations. The computation of the lowest-lying Laplacian eigenvectors is performed with the use of the ARPACK package [13]. Note that it requires no more computing effort than the usual iterative procedure.

Nevertheless, gauge-fixing remains expensive. In principle, we would like to perform a variational study over a variety of gauges. To keep computer resources within our budget, we had to limit ourselves to the two gauges above, where the links used to construct $F$ or the covariant Laplacian have been smeared 10, respectively 20 times. The price we pay for such a truncation of the variational basis is a poorer determination of the ground state. Using smeared rectangular Wilson loops yields more accurate results at cheaper cost. The specific purpose here is to exhibit the progressive mixing of the two variational states with distance.

The energy levels extracted from the matrix correlator $G(r, t)$ in eqn.(10) are linearly divergent with decreasing lattice spacing and hence do not have a continuum limit. This divergence is due to the self-energy of the static sources which, although perturbatively computable, cannot be absorbed by renormalization into a parameter of the theory. It reflects the fact that the static potential is defined only up to an arbitrary (infinite) constant, and does not itself constitute a finite physical quantity. On the other hand, the confining force and the string breaking scale are defined by energy differences such that the divergence cancels out, and these quantities do have a finite and physical continuum limit.

\section{Simulation and analysis}

In order to improve the projection properties of our operators, we employed the standard smearing algorithm 14 to obtain smeared spatial link variables of unit length, which were then used instead of the original ones in constructing the correlation functions defined above. All links in the time directions were left unsmeared such that the transfer matrix remains unaffected by our smearing procedure. As a basis of operators used in the matrix correlator eq. (10), we chose two different link smearing levels for the spatial Wilson lines and one smearing level with good projection for the clover-leaves $P$. Thus, our correlator $G(r, t)$ represents a $3 \times 3$ matrix for the gauge-invariant calculation. In 
the case of the off-axis, gauge-fixed correlators we kept only one smearing level for the unbroken string, giving a $2 \times 2$ matrix for $G(r, t)$.

The procedure we follow to diagonalize $G(r, t)$ by means of a variational calculation has been described in detail in the literature [15, 16], and its application to the calculation of the adjoint potential has already been discussed [9, 4, 7, 8]. Here we use the same algorithm as in [8] and for completeness briefly repeat the outline of the procedure. Each element $G_{i j}$ of the matrix correlator represents a correlation function which can be written as

$$
G_{i j}(r, t)=\left\langle\phi_{i}(t) \phi_{j}(0)\right\rangle
$$

where the $\phi_{i}$ 's represent a spatial gauge string of length $r$ at a given smearing level for $i=1,2$, and a two-gluelump operator at a different smearing level for $i=3$. In a temporal gauge, where all time-like links are unity, this expression describes indeed the measured correlation functions. Since all $G_{i j}$ are manifestly gauge invariant, this remains true without gauge fixing. The variational diagonalization of $G(r, t)$ consists of solving the generalized eigenvalue problem [9, 17]

$$
G(r, t) v^{i}\left(t, t_{0}\right)=\lambda_{i}\left(t, t_{0}\right) G\left(r, t_{0}\right) v^{i}\left(t, t_{0}\right), \quad t>t_{0} .
$$

From the eigenvectors $v^{i}$ one may construct the corresponding eigenstates

$$
\Phi_{i}=c_{i} \sum_{k} v_{k}^{i} \phi_{k}=\sum_{k} a_{i k} \phi_{k}
$$

which are superpositions of the string and gluelump operators used in the simulation. The constants $c_{i}$ are chosen such that $\Phi_{i}$ is normalized to unity. The diagonalized correlation matrix may then be written as

$$
\Gamma_{i}(r, t)=\left\langle\Phi_{i}(t) \Phi_{i}(0)\right\rangle=\sum_{j, k=1}^{3} a_{i j} a_{i k} \phi_{j}(t) \phi_{k}(0)=\sum_{j, k=1}^{3} a_{i j} a_{i k} G_{j k}(t),
$$

and represents the (approximate) correlation functions of the eigenstates of the Hamiltonian. To check the stability of the procedure we have performed the same calculation for $t_{0}=0, t=a, 2 a$ and obtained consistent results in both cases. The coefficients $a_{i k}$ take values between zero and one and quantify the overlap of each individual correlator $G_{i k}$ with the correlators of the mass eigenstates, $\Gamma_{i}$. They characterise the composition of the eigenstates in terms of the original operators as well as the degree of mixing between operators.

We extract the effective masses of the eigenstates $\Phi_{i}$ by assuming a single exponential decay of their correlation functions,

$$
m_{e f f}^{i}(t)=-\ln \left[\frac{\Gamma_{i}(r, t+1)}{\Gamma_{i}(r, t)}\right]
$$


The effective masses in lattice units are so heavy that the observed "plateaux" only extend over two or three $t$-values before the signal is lost in noise. In general we therefore approximate the $i$-th eigenstate of the static potential in lattice units by $a V_{i}(r / a)=$ $m_{\text {eff }}^{i}(2)$. This leaves room for a systematic error because the asymptotic plateau values might be attained only at larger $t$ and thus the energies be overestimated. In the case of the two-gluelump operator this error can be ruled out because we find it to be fully compatible with twice the single gluelump mass, for which we do see a good plateau. Only for the Wilson loop does this source of uncertainty remain. Since string breaking occurs when the energies of the broken and unbroken string are equal, overestimating the latter may lead to underestimating the string breaking distance $r_{b}$.

The size of the operator basis used here is significantly smaller than in the corresponding three-dimensional study, where up to eight operators were used $[8$. The reason simply is that our present computer resouces do not allow for a larger basis in a fourdimensional calculation. In consequence, whereas we may be fairly confident about our mass estimates for the ground state potential, the first and especially second excited states are likely to be contaminated by higher excitations. In particular, since we have only one two-gluelump operator in our basis, we are unable to compute the first excitation of the gluelump and thus cannot determine the correct ordering of the excited states for distances beyond the breaking scale, $r \gg r_{b}$. We shall come back to this point in the discussion of the results.

In order to examine the scaling behaviour of the potential, we have worked at two values of the bare coupling, $\beta=2.4$ on a $32^{4}$ lattice, and $\beta=2.5$ on a $40^{4}$ lattice. We did not find a value for $r_{0}(\beta=2.4)$ in the literature, and hence based our scaling analysis on the expression

$$
a(\beta)=400 \exp \left[-\frac{\beta \ln 2}{0.205}\right] \mathrm{fm},
$$

which describes the scaling of the fundamental representation static potential with the lattice spacing in the range of interest [18. For better comparison with other simulations we also quote our results at $\beta=2.5$ using $r_{0}(\beta=2.5)=6.39(09) a$ [19.

In three dimensions an explicit test for finite volume effects has been performed, with the finding that a spatial size $L \approx 2.4 r_{b}$ is large enough for $r_{b}$ to be free from finite size effects $[8]$. In the present work we shall find that $L>2.8 r_{b}$ for both lattice spacings and we expect no finite size effects.

At $\beta=2.4$ we employed a maximum of 40 smearing steps, whereas at $\beta=2.5$, 60 steps were necessary to reach approximate saturation in the lowering of the effec- 
tive mass of the Wilson loop. For both lattice spacings, 50 Monte Carlo sweeps were inserted between successive measurements, where one heatbath sweep was followed by four overrelaxed sweeps. For $\beta=2.4$ we collected 400 measurements for the gaugeinvariant approach and 580 for the gauge-fixed off-axis operators, whereas for $\beta=2.5$ 190 gauge-invariant measurements were sufficient to yield comparable statistical errors. The latter were estimated using a jackknife procedure, except in the case of the off-axis data, where we performed a bootstrap analysis to extract errors more reliably. We have checked the decorrelation of our measurements by observing stability of the statistical errors when the data were binned.

\section{Numerical results}

As was discussed in Section 2, we measured the mass of the gluelump as a reference to check the projection properties of the two-gluelump operator. The correlation function $G_{G}(t)$ is relatively easy to measure as it has a good signal and plateaux in the effective masses can be identified. For our two lattice spacings we extract

$$
\begin{gathered}
a M_{G}(\beta=2.4)=1.54(1), \quad a M_{G}(\beta=2.5)=1.27(1) \\
M_{G}(\beta=2.4)=2541(17) \mathrm{MeV}, \quad M_{G}(\beta=2.5)=2938(23) \mathrm{MeV} \quad\left[r_{0} M_{G}=8.12(13)\right] .
\end{gathered}
$$

In the second line the gluelump mass is given in continuum units. Recall however, that there is no physical meaning to these numbers, but the increase on the finer lattice signals the linear divergence due to self-interactions of the static source.

Figure 1 shows the three lowest states of the adjoint static potential in continuum units, as obtained from the gauge-invariant calculation at both lattice spacings. We begin by discussing the ground state. At small distances we observe the expected linear rise corresponding to an area law behaviour of the Wilson loop. The ground state then saturates rather suddenly at $r \approx 1.25 \mathrm{fm}$, maintaining an energy value of twice the gluelump mass, in accord with the expectation of string breaking. This observation is corroborated by the analysis of the operator content of the ground state, which is displayed in Figure 2 (a). In analogy with previous analyses in $(2+1)$ dimensions [8] and the fundamental potential in Higgs models [5, 6], we find the Wilson loop to have nearly full projection onto the ground state with unbroken string for $r<r_{b}$, but practically no projection onto the saturated ground state for $r>r_{b}$.

On the contrary, it maintains its projection onto the unbroken string state which is still in the spectrum but now as the first excited state. Conversely, the gluelump operator 


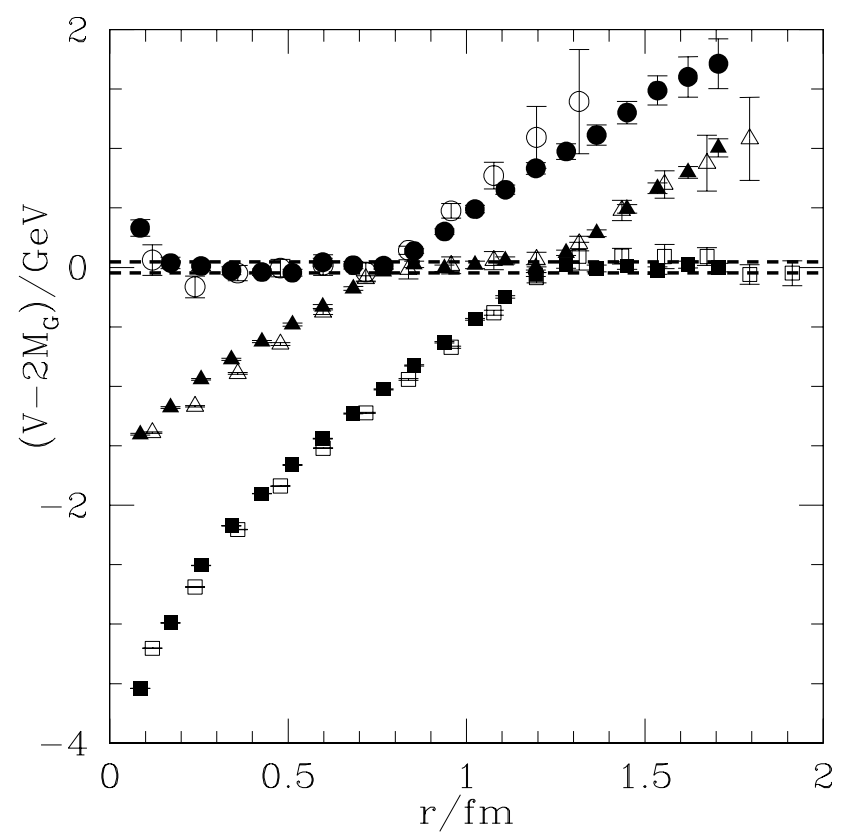

Figure 1: The adjoint potential and its first two excitations for $\beta=2.4$ (open symbols) and $\beta=2.5$ (full symbols). The dashed lines mark the error band for $2 M_{G}$.

starts out with some projection onto the ground state at small distances, which may be due to gluon exchange between the gluelumps, or simply be an artefact of the smearing procedure, since smeared gluelump operators overlap for small distances. However, this overlap with the ground state rapidly decreases with distance up to the breaking scale, where it projects fully onto the ground state, the latter now being a two-gluelump state. The crossover region with appreciable mixing between the two operator types is even narrower than was reported in the three-dimensional calculation, presumably because we are further from the continuum limit than the simulations in [8].

The ordering of states is completely analogous to the three-dimensional case [8]. In particular, at small distances the first excited state corresponds to an excitation of the gauge string, as follows from its linear rise as well as from the overlaps displayed in Figure 2 (b). It is moreover visible that it receives more contributions from the lightly smeared Wilson loop than from the highly smeared Wilson loop. Both figures furthermore show quite clearly the breaking of the excited string. In fact, the observed mixing between the Wilson loops and two-gluelump operator appears rather more pronounced than in the very rapid crossover of the ground state string breaking. We ascribe this to the fact that it happens at smaller $r$, where the Wilson loops are not as large and thus give a better 

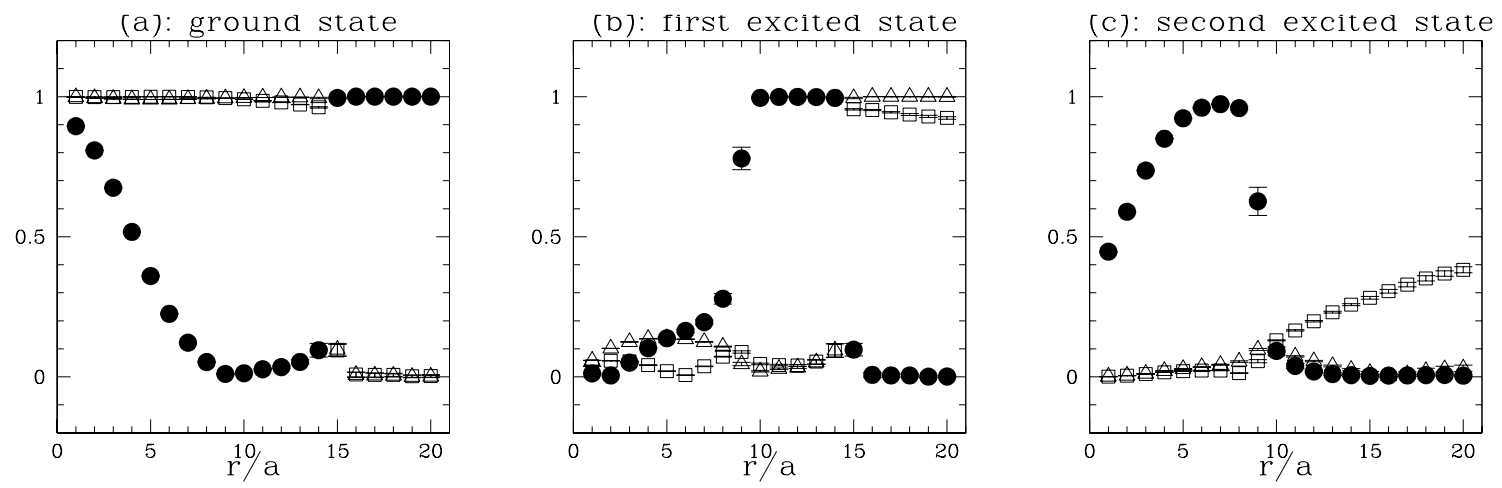

Figure 2: The overlaps $a_{i k}$ of the operators in the simulation with the lowest states of the potential, as obtained at $\beta=2.5$. Open triangles and squares: $G_{S S}$ after 40 and 60 smearings, respectively; Filled circles: $G_{G G}$.

signal for the variational calculation. After the breaking of the excited string, the first excited state of the potential is the two-gluelump state receiving its main contribution from the corresponding operator, until this level is attained by the ground state string.

For $r>r_{b}$ we do not see saturation of the linearly rising first excited state, in contrast to the three-dimensional findings [8]. This is a consequence of our limited basis consisting of just one gluelump-pair operator, which prohibits a calculation of excited gluelump states. For the same reason, we expect the excited states to be still contaminated by higher excitations.

We now follow the prescription given in [8] to determine the value of the breaking scale $r_{b}$ in continuum units. A suitable definition of $r_{b}$ for this purpose is by the distance where the string and the two-gluelump state have equal energy,

$$
\Delta \equiv E_{S}-\left.E_{G G}\right|_{r=r_{b}}=0 .
$$

To solve this equation we interpolate the energy difference between the string and the twogluelump states to the point where it vanishes. Systematic uncertainties are estimated by varying the number of points used for the interpolation. From this procedure we obtain the results

$$
\begin{aligned}
& r_{b} / a(\beta=2.4)=10.6(8), \quad r_{b} / a(\beta=2.5)=14.4(6) \\
& r_{b}(\beta=2.4)=1.27(8) \mathrm{fm}, \quad r_{b}(\beta=2.5)=1.23(5) \mathrm{fm}\left[r_{b} / r_{0}=2.25(10)\right],
\end{aligned}
$$

which exhibit satisfactory scaling behaviour.

In order to have a higher resolution in the range of string breaking and to obtain a better signal for mixing of the operators in the ground state we have performed a gauge- 
fixed calculation including off-axis correlations at irrational distances in lattice units. We have checked explicitly that the on-axis results obtained in this manner agree with those from a gauge-invariant calculation with a $2 \times 2$ matrix. It turns out, however, that Wilson loops yield a better signal than the spatial correlation of gauge-fixed sources, which need more time-like distance $t$ to attain their asymptotic behaviour. The overlaps of the two operators with the ground state in the string breaking region are shown for $\beta=2.4$ in Figure 3. Since this is the coarser lattice, the crossover in the overlaps is very rapid, even with the higher resolution. Despite their poorer quality, the data show clear evidence for progressive mixing between the two operator types, which is absent in the gauge-invariant calculation at the same $\beta$, where the ground state correlation is always composed entirely of either Wilson loop or two-gluelump operator. Finally, we remark that the $r_{b}$ one would extract from this figure is lower than the one extracted from the gauge-invariant calculation in eqn.(20), although they are statistically compatible. We nevertheless interpret this difference as a systematic effect rather than a statistical one, ascribing it to the severe limitation that a $2 \times 2$ basis poses.



Figure 3: The overlaps $a_{i k}$ of the operators with the ground state of the potential, as obtained at $\beta=2.4$ in the gauge-fixed calculation. Open squares: $G_{S S}$; Filled circles: $G_{G G}$. 


\section{Conclusions and outlook}

We have measured the adjoint static potential in 4-dimensional $S U(2)$ Yang-Mills theory. A scaling study at $\beta=2.4$ and 2.5 indicates that string breaking occurs at a distance $r_{b} \approx 1.25 \mathrm{fm}, 2.3 r_{0}$. By measuring off-axis correlations in a fixed gauge, we have observed mixing between broken and unbroken string operators, over a distance smaller than one lattice spacing $(\sim 0.1 \mathrm{fm})$. We have also resolved the breaking of the excited string, which occurs at distance $\sim 0.75 \mathrm{fm}, 1.4 r_{0}$.

Our findings are in line with the expectation that the string should break where the linearly rising part of the potential extracted from pure Wilson loop calculations intersects with the two-gluelump state [4]. The suddenness of the breaking, characterized by the near-absence of mixing noted above, as well as the ordering of the excitations are similar to what happens in $(2+1)$ dimensions [8]. In fact, the similarity is even quantitative: the string-breaking distance in units of the lightest glueball mass [20] is practically the same $\left(r_{b} m_{0^{++}} \sim 9.7\right.$ versus $10.3( \pm 1.5)$ in $\left.[8]\right)$. This suggests that identical effective mechanisms are at work.

We find no reason to expect surprises in the case of $S U(3)$. At $\beta=6.0$, the gluelump mass is known $\left(a M_{G}=1.33\right.$ [21]), and string breaking should occur at $r_{b} \sim 12.5 a$ [21], which corresponds again to a similar value of $r_{b} m_{0^{++}}$. To observe adjoint string breaking at $\beta=6.0$, a $32^{4}$ lattice should be marginally sufficient. Such a project is well within reach of better-equipped lattice groups.

The only technical difficulty here is to ascertain that broken and unbroken string operators are not completely decoupled, but do mix in the close vicinity of $r_{b}$. The mixing "window" is remarkably narrow, and it would be interesting to find the dynamical reasons for this. According to a recent suggestion [22], the suddenness of string-breaking might be caused by a qualitative change in the world-sheet spanned by the 2 static charges, which tears abruptly as the two sides are taken apart. If this change is of topological nature, one may wonder whether mixing should occur at all, or whether the string simply "snaps".

Acknowledgements The calculations for this work were performed on the NECSX4/32 at the HLRS Universität Stuttgart and on the CRAY-SV1 at ETH Zürich. O.P. also wishes to thank the ITP Universität Heidelberg for continued access to their workstations, where much of the current analysis was done. 


\section{References}

[1] C. Bernard, Nucl. Phys. B219 (1983) 341;

SESAM Collaboration (U. Glässner et al.), Phys. Lett. B383 (1996) 98;

S. Güsken, Nucl. Phys. B (Proc. Suppl.) 63 (1998) 16;

UKQCD Collaboration (C.R. Allton et al), Phys. Rev. D60 (1999) 034507;

CP-PACS Collaboration (S. Aoki et al), Nucl. Phys. B (Proc. Suppl.) 73 (1999) 216.

See review by K. Schilling, hep-lat/9909152.

[2] C. De Tar, O. Kaczmarek, F. Karsch and E. Laermann, Phys. Rev. D59 (1999) 031501;

C. De Tar, U. Heller and P. Lacock, hep-lat/9909078.

[3] G.I. Poulis and H.D. Trottier, Nucl. Phys. B (Proc. Suppl.) 42 (1995) 267; Phys. Lett. B400 (1997) 358.

S. Deldar, Nucl. Phys. B (Proc. Suppl.) 73 (1999) 587; hep-lat/9911008.

[4] C. Michael, Nucl.Phys.B (Proc.Suppl.) 26 (1992) 417.

[5] O. Philipsen and H. Wittig, Phys. Rev. Lett. 81 (1998) 4056.

[6] F. Knechtli and R. Sommer, Phys. Lett. B440 (1998) 345.

[7] P.W. Stephenson, Nucl. Phys. B550 (1999) 427.

[8] O. Philipsen and H. Wittig, Phys. Lett. B451 (1999) 146.

[9] C. Michael, Nucl. Phys. B259 (1985) 58.

[10] J.E. Mandula and M. Ogilvie, Phys. Lett. B185 (1987) 127.

[11] J.C. Vink and U.-J. Wiese, Phys. Lett. B289 (1992) 122.

[12] C. Alexandrou, Ph. de Forcrand and M. D'Elia, hep-lat/9907028.

[13] See http://www.caam.rice.edu/software/ARPACK

[14] M. Albanese et al., Phys. Lett. B192 (1987) 163; Phys. Lett. B197 (1987) 400.

[15] L.A. Griffiths, C. Michael and P.E.L. Rakow, Phys. Lett. B129 (1983) 351.

[16] O. Philipsen, M. Teper and H. Wittig, Nucl. Phys. B469 (1996) 445.

[17] M. Lüscher and U. Wolff, Nucl. Phys. B339 (1990) 222. 
[18] UKQCD Collaboration (S.P. Booth et al.), Phys. Lett. B275 (1992) 424.

[19] R. Sommer, Nucl. Phys. B411 (1994) 839.

[20] M. Teper, hep-th/9812187.

[21] UKQCD Collaboration (M. Foster and C. Michael), Phys. Rev. D59 (1999) 094509.

[22] F. Gliozzi and P. Provero, Nucl. Phys. B556 (1999) 76; hep-lat/9907023. 